\title{
OPTIMAL CHOICE OF ORDER STATISTICS UNDER CONFIDENCE REGION ESTIMATION IN CASE OF LARGE SAMPLES
}

\author{
A. Zaigrajew ${ }^{1}$, M. Alama-BuĆKO ${ }^{2}$ \\ ${ }^{1}$ Nicolaus Copernicus University of Toruń \\ ${ }^{2}$ University of Technology and Life Sciences of Bydgoszcz \\ ${ }^{1}$ Toruń and ${ }^{2}$ Bydgoszcz, POLAND \\ e-mail: ${ }^{1}$ alzaig@mat.umk.pl
}

\begin{abstract}
Let $x=\left(x_{1}, \ldots, x_{n}\right)$ be a sample from a distribution $P_{\theta}, \theta=\left(\theta_{1}, \theta_{2}\right)$, where $\theta_{1} \in R$ is a location parameter and $\theta_{2}>0$ is a scale parameter. To estimate $\theta$ strong two-dimensional confidence regions of given confidence level $\alpha \in(0,1)$ are considered. The quality of a Borel confidence set $B(x)$ is characterized by the risk function defined as $R(\theta, B)=\mathrm{E}_{\theta} \lambda_{2}(B(x))$, where $\lambda_{2}(B(x))$ is the Lebesgue measure of $B(x)$. Among confidence regions we distinguish those having the minimal risk and call them optimal. The method for construction of an optimal confidence region is well-known (see, e.g., [1]) and is based on using a pivot. Let $x_{i: n}$ represents the $i$ th order statistic of the sample $x$ for $i=1, \ldots, n$. To construct a pivot two statistics $t_{1}$ and $t_{2}$ are taken; both statistics depend on given $k \leq n$ order statistics, say $t_{1}(x)=\sum_{i=1}^{k} a_{i} x_{m_{i}: n}, t_{2}(x)=\sum_{i=1}^{k} b_{i} x_{m_{i}: n}$, where $1 \leq m_{1}<m_{2}<\ldots<m_{k} \leq n$. The case $k=2$ was considered in [4]. If $k>2$, then the problem of choosing $\left\{a_{i}, b_{i}\right\}$ is appeared. Here given $\left\{m_{i}\right\}$ the coefficients $\left\{a_{i}, b_{i}\right\}$ are taken in such a way that $t_{1}$ and $t_{2}$ are the asymptotically best linear estimators of $\theta_{1}$ and $\theta_{2}$, respectively (see, e.g., [3]). The main goal of the paper is to make the best choice of order statistics, that is the best choice of $\left\{m_{i}\right\}$, to minimize the risk function, as $n \rightarrow \infty$, under the assumptions that $m_{i} / n \rightarrow p_{i}, i=1, \ldots, k, 0 \leq p_{1}<p_{2}<\ldots<p_{k} \leq 1$. It turns out that such a problem is quite close to that considered in e.g. [2], Section 10.4. In the paper the problem of choice the value of $k$ is also discussed. Several examples of location-scale families of distributions are presented.
\end{abstract}

\section{References}

[1] Alama-Bućko M., Nagaev A.V., Zaigraev A. (2006). Asymptotic analysis of minimum volume confidence regions for location-scale families. Applicationes Mathematicae (Warszawa). Vol. 33, pp. 1-20.

[2] David H.A., Nagaraja H.N. (2003). Order Statistics. Wiley, New York.

[3] Masoom Ali M., Umbach D. (1998). Optimal linear inference using selected order statistics in location-scale models. In: Handbook of Statistics. Vol. 17, pp. 183213. North-Holland, Amsterdam.

[4] Zaigraev A., Alama-Bućko M. (2013). On optimal choice of order statistics in large samples for the construction of confidence regions for the location and scale. Metrika. Vol. 76, pp. 577-593. 